\title{
Detection of circulating tumor cells in patients with pituitary tumors
}

\author{
Gao Hua ${ }^{1,2,3,4}$, He Yanjiao ${ }^{1}$, Liu Qian ${ }^{1,3}$, Wang Jichao ${ }^{5}$ and Zhang Yazhuo ${ }^{1,2,3,4^{*}}$
}

\begin{abstract}
Background: Circulating tumor cells (CTCS) are tumor cells that have shed from a primary tumor and circulate in the peripheral blood. Recent experimental and clinical studies show that CTCs can be detected in early-stage disease.

Case presentation: We report three cases of pituitary adenoma (PA) in which tumor cells with particles were detected in the interstitial vascular compartment by transmission electron microscopy. Tumors were completely resected. Immunohistochemical analysis showed a $\beta$-catenin score of $10.5 \pm 1.5$ in the three cases with CTCs compared with $2.4 \pm 0.5$ in 24 control adenomas. The Ki-67 labeling index was $2.1 \pm 0.7$ in CTCs vs. $0.2 \pm 0.3$ in control cases $(p=0.043)$, and the $p 53$ score was $4.33 \pm 1.3$ vs. $0.31 \pm 0.17(p=0.000)$. The E-cadherin score did not differ significantly between the two groups.
\end{abstract}

Conclusions: CTCS can be detected in benign tumors such as PAs and not only in late-stage malignant tumors with apparent distant metastases. The present findings suggest that pituitary carcinomas develop from adenomas.

Keywords: Pituitary adenomas, Pituitary carcinomas, Circulating tumor cells, $\beta$-catenin, Ki-67, p53

\section{Background}

Pituitary adenomas (PAs) account for 15\% of intracranial neoplasms and are generally considered benign, although they exhibit a wide range of behaviors [1]. Many factors affect the proliferation of pituitary adenomas, such as angiogenesis [2], apoptosis [3], growth factors [4], oncogenes [5], tumor suppressor genes, and hormone receptors [6]. The most important predictor of recurrence in functioning adenomas is the basal postoperative hormone level, whereas in nonfunctioning adenomas, no single convincing factor could be identified. Age, gender, tumor size, and invasion are not related to recurrence [7]. The ability to predict tumor recurrence at the initial surgery would be helpful for decision-making regarding adjunctive therapy and to decrease morbidity. Pituitary carcinomas are very rare tumors and are defined as primary neoplasms of the adenohypophysis that undergo craniospinal and/or systemic spread [8]. Whether pituitary carcinomas develop from adenomas or regrowth remains unclear.

\footnotetext{
* Correspondence: zyz2004520@yeah.net

${ }^{1}$ Beijing Neurosurgical Insititute, Capital Medical University, Tiantan xili 6\#, Beijing 100050, China

${ }^{2}$ Key laboratory of central nervous system injury research, Tiantan xili 6\#, Beijing 100050, China

Full list of author information is available at the end of the article
}

Circulating tumor cells (CTCs) are tumor cells that have shed from a primary tumor and circulate in the peripheral blood. Recent studies show that CTCs can be detected not only in late-stage malignant tumors with apparent distant metastases, but also in early-stage disease. In addition, CTCs are potentially useful clinical markers for the diagnosis of malignant tumors and for decisionmaking regarding treatment [9]. The enumeration of CTCs is correlated with clinical outcome in prostate cancer and has been tested and used in clinical practice [10]. CTCs have been detected after chemotherapy in inflammatory breast cancer patients at high risk for relapse [11]. In the present study, we reported three cases of PA in which tumor cells with secretory granules were detected in tumor blood vessels. The expression of $\beta$-catenin, E-Cadherin, Ki-67, and p53 was analyzed in cases and in 24 control PAs to explore the mechanisms underlying recurrence and metastasis.

\section{Case presentation \\ Case 1}

A 44-year-old man was admitted to the hospital with altered visual acuity for 6 months. Magnetic resonance imaging (MRI) revealed a mass measuring $32 \times 33 \times$ $21 \mathrm{~mm}$ in the sellar region with cystic components. 
Microsurgical resection was performed by transnasal endoscopic excision of the PA. The tumor was soft and gray red with medium blood supply. On pathological examination, the tumor was negative for prolactin (PRL), growth hormone $(\mathrm{GH})$, adrenocorticotrophic hormone $(\mathrm{ACTH})$, follicle-stimulating hormone $(\mathrm{FSH})$, luteinizing hormone (LH), and thyroid-stimulating hormone (TSH). Tumor cells with particles were detected in the interstitial vascular compartment with thickening of the basement membrane (Fig. 1a). Transmission electron microscopy (TEM): The amount of mitochondria was significantly increased in the cytoplasm of tumor cells, with small round and large irregular shaped particles (Fig. 1b).

\section{Case 2}

A 25-year-old man was admitted to the hospital with intermittent headache, dizziness for 5 years, and decreased left eye vision for 1 year. Defects in the left temporal retina and partial nasal visual field were observed. MRI revealed a mass measuring $3 \times 22 \times 22 \mathrm{~mm}$ in the sellar region. Microsurgical resection was performed by transnasal endoscopic excision of the PA. The tumor was soft and gray red with medium blood supply. In the pathological examination, the tumor was positive for GH and PRL (scattered). Tumor cells with particles were detected in the interstitial vascular compartment with thickening of the basement membrane (Fig. 1c). TEM: Large particles and circular particles were observed in the cytoplasm of tumor cells. The rough endoplasmic reticulum was rich in lamellar assemblies and swelling of mitochondria was observed (Fig. 1d).

\section{Case 3}

A 40-year-old woman was admitted to the hospital with headache and amenorrhea for 6 months. MRI revealed a mass measuring $20 \times 20 \times 15 \mathrm{~mm}$ in the sellar region. Microsurgical resection was performed by transnasal endoscopic excision of the PA. The tumor was soft and gray red with medium blood supply. In the pathological examination, the tumor was positive for GH and PRL (scattered). Tumor cells with particles were detected in the interstitial vascular compartment with thickening of the basement membrane (Fig. 1e). TEM: There were round or irregular particles in the cytoplasm of tumor
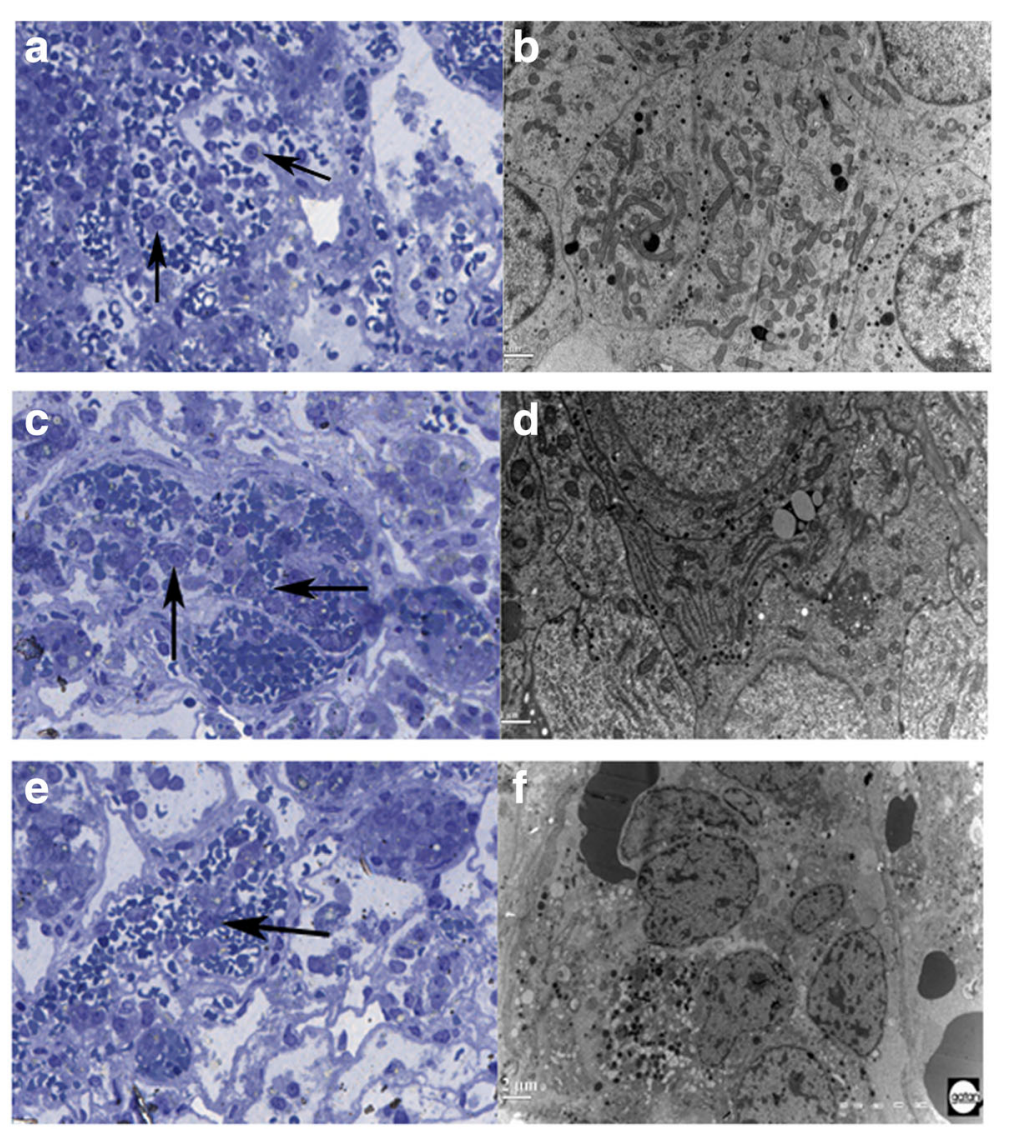

Fig. 1 CTCs in three cases were shown. Case 1: ab, Case 2:cd, Case 3: ef. ACE: Tumor cells with particles in the interstitial vascular (Arrow shown) by Azure methylene blue staining.100x. BDF: TEM 
cells. Nuclei were varied in size, with irregular shape and increased heterochromatin (Fig. 1f).

The CARE guidelines were followed when reporting these cases.

\section{Expression of the mesenchymal transition biomarkers $\beta$-catenin, E-cadherin, $\mathrm{Ki}-67$, and p53 in PA specimens} The expression of $\beta$-catenin, E-cadherin, Ki-67, and p53 was analyzed in three cases with CTC and 24 PA specimens. The specimens were fixed in $4 \%$ buffered formalin, embedded in paraffin, and incubated with anti- $\beta$-catenin (ZSGB-BIO, China), anti-E-cadherin (Abcam, UK), antiKi-67 (Leica, Germany), and anti-p53 (Leica, Germany) antibodies followed by immunodetection using the twostep polymer detection system (Polink-2 plus Kit; GBI Labs, England) and visualization with 3,3'-diaminobenzidine. Five fields at $100 \times$ magnification were randomly selected.

Staining scores were determined by a semi-quantitative system. The proportion of positive cells was scored as follows: $0:<10 \%, 1$ : $10-25 \%, 2: 26-50 \%, 3: 51-75 \%$, and $4:>75 \%$. Staining intensity was evaluated as follows:
0: no staining, 1: weak staining, light yellow, 2: moderate staining, yellowish brown, and 3: strong staining, brown. The sum score was determined by multiplying the positive proportion score by the intensity score. Immunohistochemical analysis showed that the $\beta$-catenin score in the three cases with CTCs was $10.5 \pm 1.5$ compared with $2.4 \pm 0.5$ in the control adenomas (Fig. 2a and b). The difference was statistically significant according to Fisher's exact test $(p=0.020)$. The Ki-67 labeling index (Fig. 2c and d) was $2.1 \pm 0.7$ in the three cases with CTCs compared with $0.2 \pm 0.3$ in control cases $(p=0.043)$. The p53 score (Fig. $2 \mathrm{e}$ and $\mathrm{f}$ ) was $4.33 \pm 1.3$ vs. $0.31 \pm 0.17$ in the controls $(p=0.000)$. The E-cadherin score did not differ significantly between the two groups (data not shown).

Molecular information obtained from the blood of cancer patients is an emerging and powerful research tool with great potential as a companion diagnostic factor for patient stratification and monitoring [12]. We detected CTCs with secretory granules in the vessels of patients with PAs. Recent experimental and clinical studies show that CTCs can be detected not only in late-stage malignant tumors with apparent distant metastases, but also in

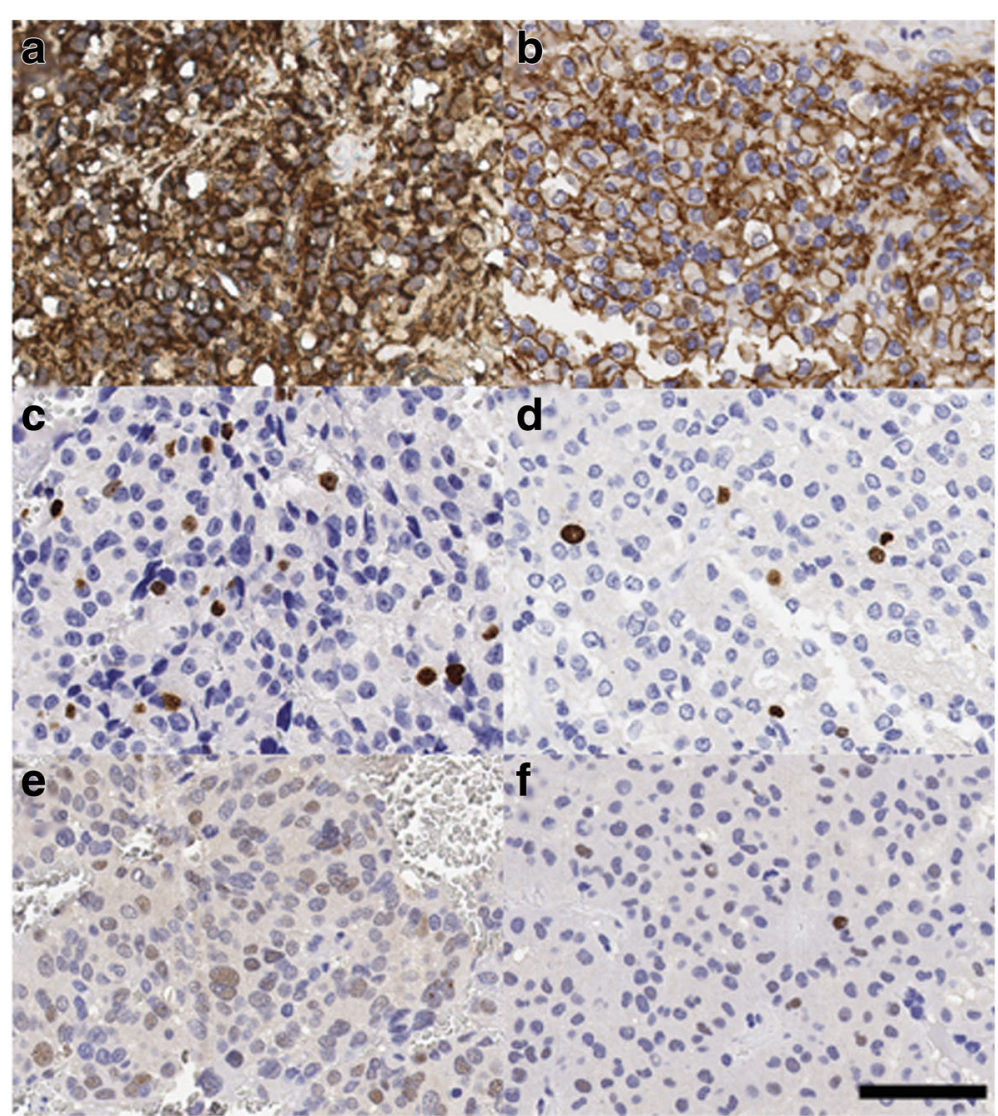

Fig. $2 \mathrm{HC}$ analysis of the $\beta$-catenin and Ki67 expression. a $\beta$-catenin expression in PAs with CTCs. b $\beta$-catenin expression in control PAs. $\mathbf{c}$ Ki67 expression in PAs with CTCs. d Ki67 expression in control PAs. e p53 expression in PAs with CTCs. $\mathbf{f}$ p53 expression in control PAs. Bar $=60 \mu \mathrm{m}$ 
early-stage disease [13]. Furthermore, CTCs were identified as prognostic factors in several solid tumors such as colorectal, pancreatic, gastric, breast, and genitourinary cancers [14].

Pituitary carcinomas are classically defined as pituitary tumors with subcranial, brain, or systemic metastases [15]. In general, pituitary carcinomas are associated with poor prognosis as therapeutic options are limited. Whether pituitary carcinomas develop from adenomas or if they occur de novo remains unclear; in addition, the effect of hormonal subtypes on tumor aggressiveness, treatment outcomes, and prognosis has not been elucidated [16]. The present findings provide indirect evidence supporting that pituitary carcinomas develop from adenomas.

Data from a retrospective case-control study of 410 patients led to the classification of PAs according to tumor size and immunohistochemical type, and the assessment of invasion and proliferation provides prognostic information for predicting postoperative disease-free outcome or recurrence/progression status [17]. Epithelialmesenchymal transition (EMT) is widely recognized as a key process associated with malignant transformation, and the $\mathrm{Wnt} / \beta$-catenin pathway plays a regulatory role in EMT because of its involvement in maintaining epithelial integrity and tight adhesion junctions [18]. $\beta$-catenin, an important effector in the Wnt signaling pathway, is important for cell-cell adhesion. The E-cadherin/ $\beta$-catenin complex functions in intercellular junctions that promote cell adhesion [19]. We found that the Ki-67 LI was $>10 \%$, and the $\beta$-catenin positivity rate was $>90 \%$ (score: $10.5 \pm$ $1.5)$ in cases with CTCs. However, there was no statistically significant difference in the level of E-cadherin between the two groups. Abnormal $\beta$-catenin expression was correlated with more advanced disease stage, and as a consequence, with poor outcome in gastric cancer [20]. Nuclear E-cadherin is common in nonfunctioning PAs and a subset of growth hormone-secreting adenomas, in which it is associated with tumor size and invasion [21].

Ki-67, a marker of cellular proliferation, has been studied extensively in pituitary neoplasias [22]. It is of relevance to various clinicopathological parameters, including tumor subtype, size, invasiveness, and recurrence, as well as patient age and sex [23]. In malignant prolactinoma, the Ki-67 index of the tumor was $24.8 \%$, and $60 \%$ of tumor cells were positive for p53 [24]. In another study, pathology revealed a high Ki-67 index in metastatic tumor tissues in two cases $(24.2 \%$ and $10 \%)$ with positive p53 staining in one pituitary carcinoma [25]. In the current tumor classification of the World Health Organization, the definition of an atypical PA includes a Ki-67 labeling LI $>3 \%$ and extensive p53 positivity. In fact, PAs with Ki-67 expression $\geq 1.5 \%$ showed a higher recurrence risk and a worse disease-free survival compared with those with Ki-67 expression $<1.5 \%[26]$.

\section{Conclusion}

Tumor recurrence is a significant clinical problem in PAs in which patients are asymptomatic because disseminated cells that become dormant are undetectable by clinical tools. Patients with CTCs may benefit from aggressive treatment such as radiotherapy and close surveillance.

\begin{abstract}
Abbreviations
ACTH: Adrenocorti cotrophic hormone; CTCs: Circulating tumor cells; EMT: Epithelial-mesenchymal transition; FSH: Follicle-Stimulating Hormone; GH: Growth hormone; Ki67 LI: Ki67 labelling index; LH: Luteinizing hormone; MRI: Magnetic resonance imaging; PAs: Pituitary adenomas; PRL: Prolactin; TEM: Transmission electron microscope; TSH: Thyroid-stimulating hormone
\end{abstract}

\section{Funding}

This work was supported by Beijing Natural Science Foundation of China (7162035) and Beijing high-level talent plan (2015-3-040).

\section{Availability of data and materials}

We agree that all datasets on which the conclusions of the manuscript rely to be either deposited in publicly available repositories (where available and appropriate) or presented in the main paper or additional supporting files, in machine-readable format (such as spreadsheets rather than PDFs) whenever possible. Beijing Natural Science Foundation of China: collection and interpretation of data; Beijing high-level talent plan: writing and revising the manuscript.

\section{Authors' contributions}

$\mathrm{GH}$ design and writing, $\mathrm{HYJ}$ the experiment of transmission electron microscope, LQ staining; WJC staining and operation, ZYZ scanning the slides and operation. All authors read and approved the final manuscript.

Ethics approval and consent to participate

Informed consent was obtained from all individuals and ethical approval was obtained from the Institutional Review Board of Beijing Tiantan Hospital Affiliated to Capital Medical University (KY2013-015-02).

\section{Consent for publication}

Written informed consent was obtained from the patient for publication of this case report and any accompanying images. A copy of the written consent is available for review by the Editor of this journal.

\section{Competing interests}

The authors declare that they have no competing of interest.

\section{Publisher's Note}

Springer Nature remains neutral with regard to jurisdictional claims in published maps and institutional affiliations.

\section{Author details}

${ }^{1}$ Beijing Neurosurgical Insititute, Capital Medical University, Tiantan xili 6\#, Beijing 100050, China. ${ }^{2}$ Key laboratory of central nervous system injury research, Tiantan xili 6\#, Beijing 100050, China. ${ }^{3}$ Center of Brain Tumor, Beijing Institute for Brain Disorders, Tiantan xili 6\#, Beijing 100050, China. ${ }^{4}$ China National Clinical Research Center for Neurological Diseases, Tiantan xili 6\#, Beijing 100050, China. ${ }^{5}$ Department of Neurosurgery, Xinjiang Uygur Autonomous Region People's Hospital, Xinjiang 830000, China.

Received: 3 May 2016 Accepted: 21 February 2018

Published online: 27 March 2018

References

1. Yu R, Melmed S. Pathogenesis of pituitary tumors. Prog Brain Res. 2010; 182(3):207-27.

2. Turner HE, Nagy Z, Gatter KC, Esiri MM, Harris AL, Wass JA. Angiogenesis in pituitary adenomas - relationship to endocrine function, treatment and outcome. J Endocrinol. 2000;165(2):475-81.

3. Sambaziotis D, Kapranos N, Kontogeorgos G. Correlation of BCl-2 and Bax with apoptosis in human pituitary adenomas. Pituitary. 2003;6(3):127-33. 
4. Spada A, Lania A. Growth factors and human pituitary adenomas. Molec Cell Endocrinol. 2002;197(3):63-8.

5. Jiang $X$, Zhang $X$. The molecular pathogenesis of pituitary adenomas: an update. Endocrinol Metab (Seoul). 2013;28(4):245-54.

6. Antonio S, Giuseppe M, Andrea R, Esposito V, Jaffrain-Rea ML, Delfini R. Biochemical remission and recurrence rate of secreting pituitary adenomas after transsphenoidal adenomectomy: long-term endocrinologic follow-up results. Surg Neurol. 2007;68(5):513-8.

7. Roelfsema F, Biermasz NR, Pereira AM. Clinical factors involved in the recurrence of pituitary adenomas after surgical remission: a structured review and meta-analysis. Pituitary. 2012;15(1):71-83.

8. Pernicone PJ, Scheithauer BW, Sebo TJ, Kovacs KT, Horvath E, Young WF Jr, et al. Pituitary carcinoma: a clinicopathologic study of 15 cases. Cancer. 1997;79(4):804-12.

9. Andergassen U, Zebisch M, Kölbl AC, Köniq A, Heublein S, Schröder L, et al. Real-Time qPCRbased detection of circulating tumor cells from blood samples of adjuvant breast cancer patient: a preliminary study. Breast Care (Basel). 2016;11(3):194-8.

10. Galletti G, Portella L, Tagawa ST, Kirby BJ, Giannakakou P, Nanus DM. Circulating tumor cells in prostate cancer diagnosis and monitoring: an appraisal of clinical potential. Molec Diagnos Ther. 2014;18(4):389-402.

11. Hall CS, Karhade M, Laubacher BA, Kuerer HM, Krishnamurthy S, DeSnyder S, et al. Circulating tumor cells and recurrence after primary systemic therapy in stage III inflammatory breast cancer. Jnci J N Cancer Ins. 2015;107(11).

12. Pantel $\mathrm{K}$, Alix-Panabières $\mathrm{C}$. Functional studies on viable circulating tumor cells. Clin Chem. 2016:62(2):328-34.

13. Allard WJ, Matera J, Miller MC, Repollet M, Connelly MC, Rao C, et al. Tumor cells circulate in the peripheral blood of all major carcinomas but not in healthy subjects or patients with nonmalignant diseases. Clin Cancer Res. 2004;10:6897-904.

14. Alix-Panabières C. EPISPOT assay: detection of viable DTCs/CTCs in solid tumor patients. Recent Results Cancer Res. 2012;195:69-76.

15. Raverot G, Burman P, McCormack A, Heaney A, Petersenn S, Popovic V, et al. European Society of Endocrinology. European Society of Endocrinology Clinical Practice Guidelines for the management of aggressive pituitary tumours and carcinomas. Eur J Endocrinol. 2018;178(1):G1-G24.

16. Colao A, Ochoa AS, Auriemma RS, Faggiano A, Pivonello R, Lombardi G. Pituitary carcinomas. Front Horm Res. 2010;38(38):94-108.

17. Raverot G, Vasiljevic A, Jouanneau E, Trouillas JA. Prognostic clinicopathologic classification of pituitary endocrine tumors. Endocrinol Metab Clin N Am. 2015;44(1):11-8.

18. Zhao S, Bellone S, Lopez S, Thakral D, Schwab C, English DP, et al. Mutational landscape of uterine and ovarian carcinosarcomas implicates histone genes in epithelial-mesenchymal transition. PNAS. 2016;113(43):12238-43.

19. Chaw SY, Majeed AA, Dalley AJ, Chan A, Stein S, Farah CS. Epithelial to mesenchymal transition (EMT) biomarkers-E-cadherin, beta-catenin, APC and vimentin-in oral squamous cell carcinogenesis and transformation. Oral Oncol. 2012:48(10):997-1006.

20. Bartolomeo MD, Pietrantonio F, Pellegrinelli A, Martinetti A, Mariani L, Daidone MG, et al. Osteopontin, E-cadherin, and $\beta$-catenin expression as prognostic biomarkers in patients with radically resected gastric cancer. Gastric Cancer. 2016;19(2):412-20.

21. Elston MS, Gill AJ, Conaglen JV, Clarkson A, Cook RJ, Little NS, Robinson BG, Clifton-Bligh RJ, McDonald KL. Nuclear accumulation of e-cadherin correlates with loss of cytoplasmic membrane staining and invasion in pituitary adenomas. J Clin Endocrinol Metab. 2009;94(4):1436-42.

22. Vasiljevic A, Jouanneau E, Trouillas J, Raverot G. Clinicopathological prognostic and theranostic markers in pituitary tumors. Minerva Endocrinol. 2016:41(3):377-89.

23. Salehi F, Agur A, Scheithauer BW, Kovacs K, Lloyd RV, Cusimano M. Ki-67 in pituitary neoplasms: a review-part I. Neurosurgery. 2009:65(3):429-37.

24. Phillips J, East HE, French SE, Melcescu E, Hamilton RD, Nicholas WC, et al. What causes a prolactinoma to be aggressive or to become a pituitary carcinoma? Hormones (Athens). 2012;11(4):477-82.

25. Carlos KM, David C, Steven GW, Christopher HC, Anita M, Paul DB, et al. Radiotherapy with concurrent temozolomide for the management of extraneural metastases in pituitary carcinoma. Pituitary. 2016;19(4):415-21.

26. Chiloiro S, Doglietto F, Trapasso B, lacovazzo D, Giampietro A, Di Nardo F, et al. Typical and atypical pituitary adenomas: a single-center analysis of outcome and prognosis. Neuroendocrinology. 2015;101(2):143-50.

\section{Submit your next manuscript to BioMed Central and we will help you at every step:}

- We accept pre-submission inquiries

- Our selector tool helps you to find the most relevant journal

- We provide round the clock customer support

- Convenient online submission

- Thorough peer review

- Inclusion in PubMed and all major indexing services

- Maximum visibility for your research

Submit your manuscript at www.biomedcentral.com/submit
Biomed Central 